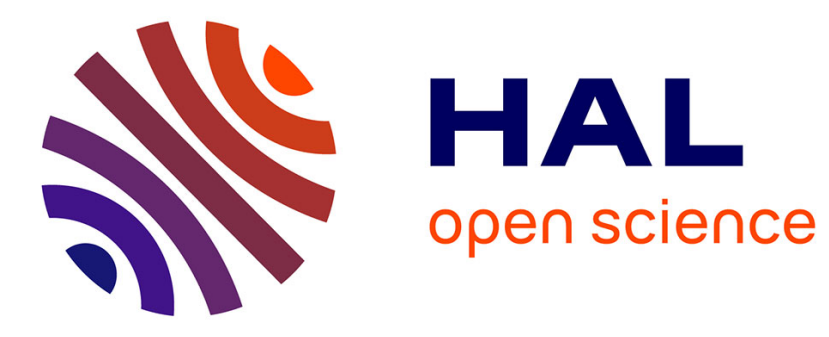

\title{
Kalman Filter Contributions Towards Document Segmentation
}

Charles Queguiner, Jean Camillerapp, Ivan Leplumey

\section{To cite this version:}

Charles Queguiner, Jean Camillerapp, Ivan Leplumey. Kalman Filter Contributions Towards Document Segmentation. ICDAR 1995 Third International Conference on Document Analysis and Recognition, Aug 1995, Montreal, Canada. pp.765-769. hal-00763395

\section{HAL Id: hal-00763395 \\ https://hal.inria.fr/hal-00763395}

Submitted on 10 Dec 2012

HAL is a multi-disciplinary open access archive for the deposit and dissemination of scientific research documents, whether they are published or not. The documents may come from teaching and research institutions in France or abroad, or from public or private research centers.
L'archive ouverte pluridisciplinaire HAL, est destinée au dépôt et à la diffusion de documents scientifiques de niveau recherche, publiés ou non, émanant des établissements d'enseignement et de recherche français ou étrangers, des laboratoires publics ou privés. 


\title{
Kalman Filter Contributions Towards Document Segmentation
}

\author{
Ivan Leplumey (t), Jean Camillerapp (+), Charles Queguiner (*)
}

\author{
(+) INSA-IRISA 20, av. des Buttes de Coesmes - 35043 RENNES CEDEX - FRANCE \\ (*) IFSIC-IRISA Campus de Beaulieu-35042 RENNES CEDEX - FRANCB \\ \{Ivan.Leplumey, Jean,Camillerapp, Charles.Queguiner\}@irisa.fr
}

\section{Abstract}

One way of tackling document analysis is to spllt the document into different layers and then to find a segmentation for each one. The straight line is often one of the basic elements of the layers making up a document. The detection of these segments is often disturbed by the superposillon of different layers in the same area. Therefore we developed a segment extractor, which processes masked areas, dis. continutities and even curvatures. After showing in our prevlous work, the interest of such a system in binary image andlysis, our aim was to show' the method could be genter. alized to grey level intages, by taking inlo account pixel lu. minosity as one of the parameters. The Kalman filter method combined with an adequate control scheme, allows the pooling of local observations, when their position. width and luminosity are coherent. The proposed model gives an imterresting scheme to manage drawings masking each other (eg. cross-lines).

\section{Introduction}

Document segmentation aims at making easier the doc. ument interpretation. Losating different kind of information in a document global contents is a part of document segmentation. Some documents, such as maps, musical scores, written checks can be seen as a superposition of multiple information layers [1]. For example, we can easily discem two layers in the image of a check: the background layer (printed pajer, base lines, preprinted information) and the superimposed handwritten characters, Most people are working on binary images but, as we think the luminosity given by grey levels could improve the segmentation process, we propose to work on grey level images.

To achieve the aralysis of a document, we try to separate the different components to recognize them individually. Multi layer superimpositions bring about difficulties. The "linear drawings" are often one of the background elements. They often interfere with handivriting on preprinted documents (checks, forms, ...). In our study, we tried to discover those linear drawings (also called "segments").
- "Line drawing" (also called "segmene") derinition

A linear drawing is defined as an alignment of pixels. Bach of them being chosen as a representative point of a real observation extracted from the image (see section 3). It is necessary to define some requested properties.

- (1) meaningfulness of the neighborhood : an alignment of isolated points is not considered as a linear drawing.

- (2) possible existence of discontinuities : in is useful to allow locally an absence of points, due to the quality or the nature of the extracled object (dotted linear drawings).

Discogtinuity (noise)

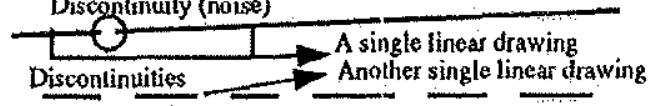

- (3) Use of the different parometers related to each representative point, such as thickness and brightness of the corresponding observation (for grey level images).

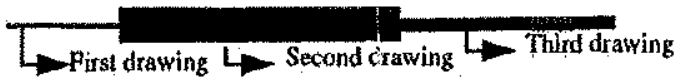

- (4) extraction of segments of different sizes, (ranging from a few points to several hundred representative points).

- (5) possibility of a drawings superimposilion, e.g. :

- staves in a musical scorc covered by quavers bars,

- base lines on a written check.

This superimposition characteristic results from the document layers theory.

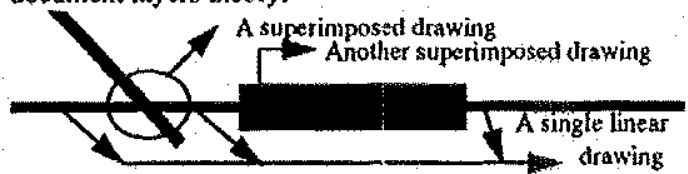

- (6) Lastly, possibility to include a possible "curvature" as a "straight segment" although it may be seen contradictory to the "linear drawing" notion. Locally, we can observe a straight line but, globally, this "straight" segment is slightly curved, and we can decide to keep this or not.

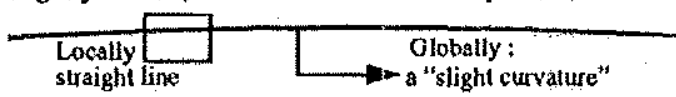


- Linear drawing extraction mefluods

Two methods are commonly used to solve the straight segment detection problem.

- The first : Hough transform [2] does not respect (6).

- The second : includes a thinning stage followed by an iterative polygonal matching, but does not respect (2) (\$).

- That's why we propose a method which respects all the six previously described propertics. It locally works on linear drawing hypotheses which are confremed or not during the analysis of the image. The used measures do not only concern the spatial location of the drawing, but also other characteristics (thickness and grey level).

Our method included two levels. The first level contained a prediction/verification tool : the Kalman filter [3]. The second level was a control layer permitting to interpret the expected cases (discontinuities, crossings). In a similar way, Jean-Pierre Veran [6], also used the Kalman filter, to follow roads in aerial grey level images.

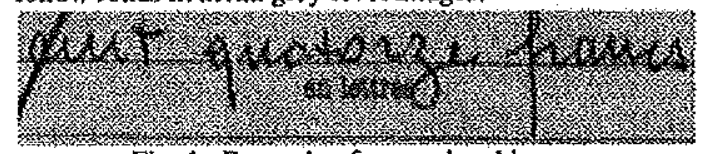

Fig. 1 : Example of a grey level image

\section{Kalman filtering}

What we were trying to detect was a series of nearly horizontal aligned observations (base lines on checks).

2.1 General principle of prediction/verification The basic hypothesis is that any object can be characterized by a state $c_{k}$ varying in relation to a scale, usually time. The method allows to follow the evolution of this state to estime it and also to predict it. For that purpose the method used a recursive linear estimator which according to the known past of a state $e_{k}$, can predict ils evolution.

$e_{k}=$ linear estimation $\left(e_{k-1}, e_{k-2}, e_{k-3}, \ldots, e_{0}\right)$.

After predicting this stage, the next step is to verify it by examing the actual current state of the object and correcting the estimator by taking into account the error made between the prediction and the measured observation.

Accepting errons and correcting the estimator by taking those errors into account gives this principle all his power to manage the state of an object. However, it implies knowing how the state of the object can vary with time, i.e. having a model of this object.

\subsection{Kalman filter equations}

The Kadman filter formalizes accurately the prediction/ verification principle. This is a recursive linear estimator which offers the advantage of taking into account calculated errors between measurements and estimations.

Those errors are automatically incorporated into the model to improve future estimations.

This method uses two models [5] :

$\rightarrow$ a stale model $S$, varying with time and
- a model of measure $X$, deducted from $S$.

Botti the models $S$ and $X$ are represented by a vector. $S$ cannot be direclly known and we are trying to estimate it. Evolution state equation :

$S(k)=A(k-1) \cdot S(k-1)+W(k-1)$

where $W$ stands for the model error, characterized by a zero mean and a variance $V w$. $A$ is a matrix characlerizing the evolution of the vector $S$.

Measure equation :

$$
\mathrm{X}(\mathrm{k})=\mathrm{C}(\mathrm{k}) \cdot \mathrm{S}(\mathrm{k})+\mathrm{N}(\mathrm{k})
$$

where $C$ is a matrix used to deduct the measure vector $X$ from the state vector $S$, and where $N$ stands for the measure noise with a zero mean and a variance $Y n$.

Besides, it is important to note that $W$ and $N$ are supposed to be uncortelated.

To find the Kalman filter equations, see [5],

\section{Observation extracted from an image}

To apply this theory, it is necessary to define shat kind of object we are sludying. We have to define its state, and its evolution model in the course of time (here columns of the image). We are searching for "observation" alignments in the columns. These pooled observations will define the linear drawings that are the objects we are looking for in the document image. The state of an object will be determined from parametcrs, chosen for the object model definition,

First of all, we had to define the observations looked for in the image. Vincent Poulain d'Andecy in [4] defined observations in binary images, we propose to extend this definition to grey level images.

It must be remembered that we were trying to extract horizontal base lines in grey level images of checks (Fig. 1). Those lines can be considered as dirk thin objects laid on a clear background. This characteristic determined the observation model we were going to search for in the image. A cross-section of a thin horizontal object has a typical luminosity profile pattem, that we tried to find in the image matrix. We sought in each column, a sequence of points of decreasing luminosity, followed by another point sequence of increasing luminosity. Several parameters, which corresponded to the luminosity and position in the column of these remarkable points of the observation, were associated to each detection and recorded as follows :

- begin point of decreasing luminosity (bln, blumi)

- medium point (min,mluni) : last polnt of decreasing

luminosity and first point of the increasing luminosity

- Last point of the increasing luminosity (lin, llumi).

As the two point positions, bin and lln, were too noise sensitive, we added two new points (milln and mi2ln) which had a better position stability. Those points, are located respectively half-way dowil and lialf-way up the luminosity curves (see Fig. 2). They corresponded fairly well to points selected by edge detectors. 


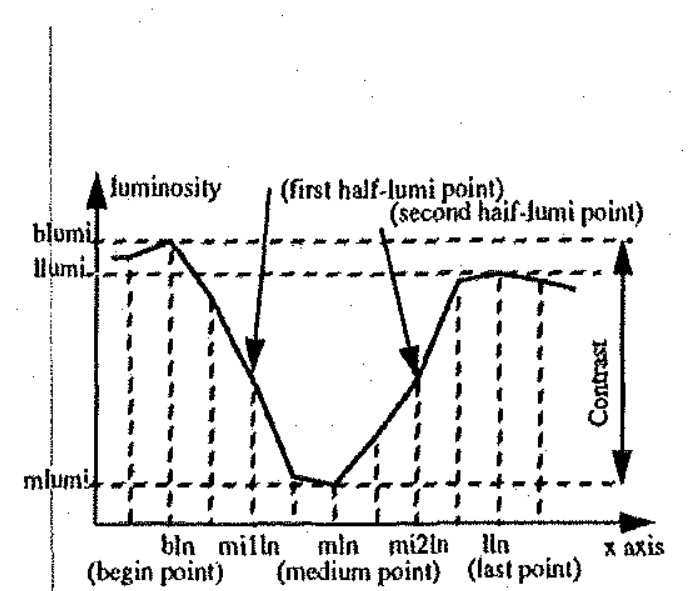

Fig. 2: An observation (luminosity curye profile) and the eighl associated parameters

As a result, an observation was defined by eight paraneters. Dependiny on the rest of the application, they are not all necessary. The techrique we used only divided one column of an image into a succession of intervals that could be parts of a linear drawing. We noticed that a great number of those observations corresponded to noise in the image, that's why a selection process was necessary. To achieve that we associated to each observation, we calculated a contrast (max (blumi, llumi) - mitumi). Every observation with a contrast below a threshold was discarded.

The observations comesponding to noise that were not eliminated by the previous thresliolding were noneritical for the method. The obtained results are robust to this threshold usage. The final decision to consides an observation as interesting or not was based on a broader context of histeresis thresholding. Figure 3 shows the observations (Fig $3 \mathrm{~b}$, in black) obtained from the original image (Fig 3 a) with a contrast threshold of 30 grey levels.

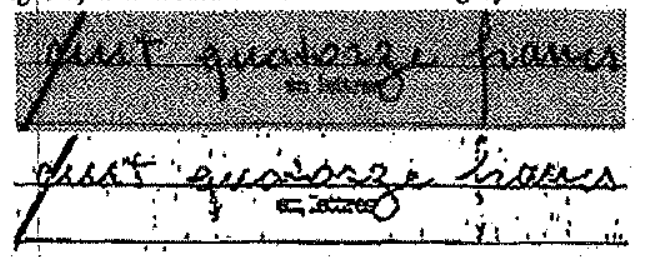

Pig. 3 : a-Original image, $b$ - extracted observations.

The "linear drawing" object we were looking for, was finally characterized by four parameters :

- position (in the column)

- slope (computed along the drawing)

- thickness (calculated with the observations)

- Luminosity (given by the observations).

A lirst step consits in extrateting obscrvations for a column $\mathrm{k}$ of the image. A second step consists in a prediction from existing objects hypotheses, Finally, a difficult problem temains to be solved the choice of the actual observations which will be used for verilication purposes. One of the Kalman filter's assets is to automatically carry out paramelers to make this choice.

\section{Application to segment detection: state and measure equations}

As we are looking for straight lines in an image, following section 3 conclusions, we propose a state vector (quadnplet) $S=$ (position, slope, thickness, luminosity).

The slate cquation showing the object evolution then is :

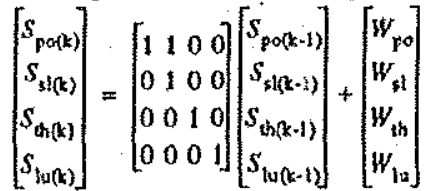

$$
S(k)=A(k-1 \quad S(k-1)+W(k-1)
$$

The measurement equation matches the studied obser vation and the state model of the segment at instant $k$. Hence the following equation:

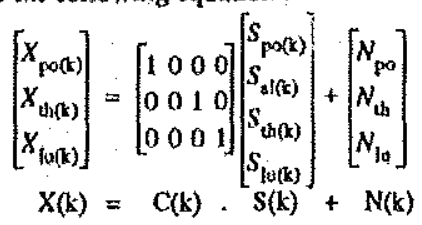

The vectors $W$ and $N$ are only characterized by a priori fixed yariances,

Final equations can easily be obtained by using matrices $A$ and $C$ in the general equations of the Kalman filter [5]. We shall not develop the choice of error pammeters which are linked to both what we are looking for and what we are measuring. Thus, if we decide to refuse a "curved line segment" (property (6)), we choose a position model with errors $\left(W_{\text {position, }} W_{\text {pente }}\right)$ valued close to zero.

The tool we are presenting, allows us, if a linear drawing has been expected up to column $(k \cdot 1)$ to :

- predict the characteristies of the observation expected for column $k$ :

- evaluate associated error predictions, depending on model errors and observation trors (image noise);

- take into account this observation (matched with a seg. ment) for the calculations concerning next columns.

\section{5 "Segment hypothesis" control scheme}

The segment hypothesis control scheme consists of a loop, marked by the column number in the image. This loop contains the four following actions :

- for each hypothesis, a prediction stago, updating each Kalman filter (one per segment hypothesis);

- a matching stage, also called verification stage, matching hypotlesis and observations ;

- a new hypothesis creating stage (when an observation didn't match an existing segment hypothesis) ;

- a hypothesis stopping stage (when no observation matched one hypothesis) ; to keep it for future interpretation or to erase it when not surficiently confimed. 


\subsection{Matching observations' segment hypotheses -Principle}

The most difficult point of the control scheme was matching working hypotheses with current column observations. For each hypothesis we had to find, the corre sponding observation, if it exists, anong all those extracted from the image column. A way to do this was to calculate for each observation, its distance from all hypotheses and to choose those within a good distance range.

Knowing $\sigma_{\text {Thickness: }} \sigma_{\text {Luminosity }}$ and $\sigma_{\text {position }}$ given by Kalman filter's covariance matrix [5], allowed us to use the Mahalanobis distance using the three parameters : thickness, luminosity, position. The $\chi^{2}$ test, used with three degrees of freedom, allowed us to define a distance value for the matching. However, we had to cope with three cases :

- one observation matched one hypothesis :

- many observations matched one hypothesis ;

- no observation matched a hypothesis.

\section{- Multiple matching}

In the case of multiple observations matching one hy pothesis, we choose to match it with the nearest observation in terms of Mahalanobis distance, This is also known as NNSF filler (Nearest-Neighbor Standard Filter).

-Absence of matching

When no observation existed within the given distarce range : was it a total absence of observations in the predicted area, or was it only because we were getting through a superimposed object?

In the case of actual absence of observations, our choice was to add the predicted observation to the Kalman fitter, delaying the decision for a certain time.

The second case, getting through a superimposed object, had to be better defined. The semantics of the image helped us to explain this. We were working on check images where the handwriting is darker than the preprinted base lines are. When those two kinds of information superimpose, only the handwriting is apparent. The fuminosity parameter of the observation extracted from the innage could be very different from the predicted one, so there was no observation matching, despite the fact that the position parameter was "good". That's why we considered that this real observation was crossed by "another" hypothesis :

- if the neal observation corresponded to the predicted

- if the thickntess was larger:

- if the luminosity was darker.

This crossing idea is fundamental since in allows us to "wail" before discarding or terminating a seginent hypothesis. As a matter of fact. when a clear linear drawing was covered by bandwriting, we waited until we found an observation which matched or did not match before deciding the hypothesis future. Moreover, this idea can help us to improve image segmentation by providing us some expla. nations about specific luminosity proftle (when two straight lines cross each other). We could then predict the luminosity profiles of soveral hypotheses that cross each other and then deduct associated observations.

\subsection{Creation of a segment hypothesis}

The conditions to create a new segment hypothesis are simple : every observation being unmatched with an existing hypothesis generates a new hypothesis. Knowing that a Kalman filter is associated with each hypothesis, it is necessary to use a stopping strategy for those new hypotheses otherwise the required computing time will rise.

\subsection{End of a segment hypothesis -Principle}

To prevent an increase in computing time, it is necessary to keep only promising hypotheses, those that still matched observations, and to get rid of the others.

-Lack of observation matching for a certain time

Hypothesis that do not match observations for a certain time will be discarded. The future of a hypolhesis depends on how long it renains active. If its life was long enough, the hypothesis is stored in a data structure for later interpretation, if nol it is "dropped" except of coure if the hypoth. esis is in a crossing state.

- Hypothesis initiation

To better control the "living" hypotheses, we used an in duction threshold (minimum two first real observations).

\section{- Hypothesis synonymy}

When two different hypothesis matched the same observalton, the program affected this observation to those two different hypotheses. If this was the case for a certain time. we used the Mahalonobis distance to check they were not similar. If the distance is small enough we considered those two hypotheses as syronyms. The crossing case, involving two straight lines having different directions, was sorted out by comparing their respective slope. The observation could be linked to both hypotheses, but the slope would keep its Mahalonobis distance above a threshold. This allowed those two different hypotheses to survive.

\section{Results}

The obtained resuits show the method's interest. Figure 4 shows extracted linear drawings (longer than 10 pixels) with the described Kalman filter based on position, thickness and luminosity coherence. It is obvious that the two base lines of the check are detected, though they are superimposed with checkbars and handwriting.

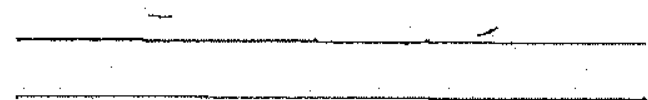

Fig. 4 : The detected tinear drawings.

The detected observations, crossed by several hypotheses 
are shown in white (superimposed with the original image) (Fig. 5), In those regions segmentation will be improved

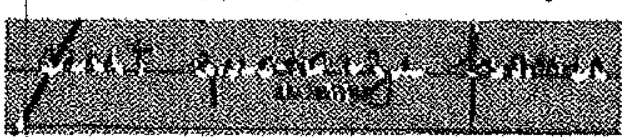

Fig. 5 : Observations (white) crossed by many hypotheses The following figures show the obtained layers partition (notes, quaver bars, ...) from a musical score binary image:

The stave lines have been removed (original Fig. 7a):

- by the common technique in two stages.

- firstly locating the different lines.

- secondly removing the observations with the right location and the right thickness, independently of other context (defaults: see circles on Fig. 6a).

- by our cooperative technique between low and high levels. The results show a good shape along the edges.

,

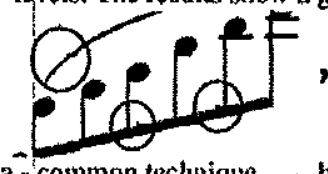

common tecliniquo

- alman fitter method. (simall angles left) (smoother shape)

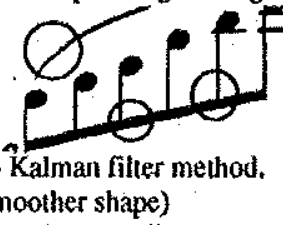

Fig. 6: The image without the stave lines.

On the following image, the quaver bar, part of a layer, has been removed, without distortion for the stave lines, thanks to the proposed Kalman filter method (Fig. 7b),

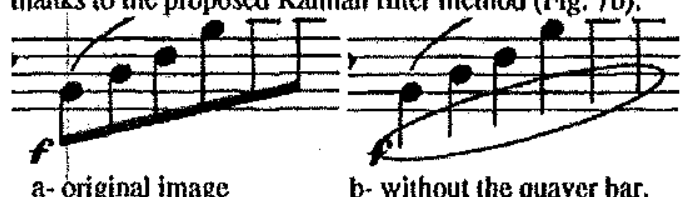

Fig. $7:$ The image without the quaver bar.

The last figure shows our grey level check image, after removing the base lines, We can notice that the oblique tine is not erased. The crossing zones are conserved with some aspect defects as square edges instead of round edges (see circles in Fig. 8).

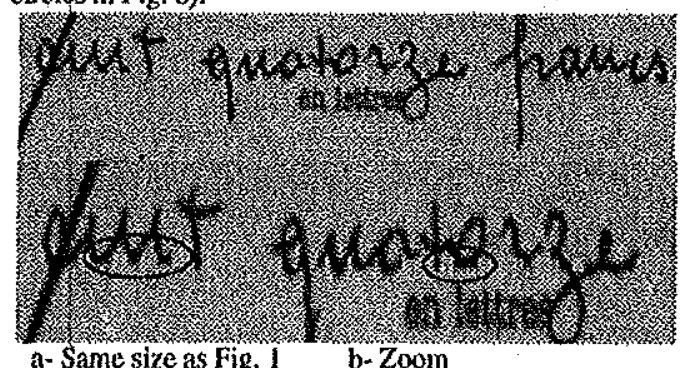

Fig. 8 : The check after elimination of the two base lines

Computing time for this inage ( 730 columns $\times 100$ lines) is about half a second. The average number of linear drawings hypotheses on this image is four per column.

\section{Conclusion}

The presented method whose purpose is to extract linear drawings has some characteristics that can be considered as drawbacks :

- the results are sensitive to the scanning orienlation :

They can present some small "pixel level" differences.

- the detection depends on the orientation of the linear drawings (best with slope botween $+45^{\circ}$ and $-45^{\prime}$ ):

Nearly vertical linear drawings cannot be detected by a column oriented scan. A line oriented scan solve this.

- the method uses a great number of parameters :

Nevertheless, it is very easy to use and also very robust. As a matter of fact, the error parameters for the model and for the observations (position, thickness, lumi) are managed by the model controlling the Kalman filter.

We consider the following points as advantages:

- The method can easily accept new parameters (see section 4).

The luminosity and thickness were added to our initial Kalman filter model (for biriary images). Thanks to good parameter usage, this method is very flexible. It can even accept some slight curves in detected linear drawings, by relaxing the model error parameter.

- the ntethod manages to widen its decision context.

By integrating compatible observations provided during the scan (see section 5), there is a cooperation between local analysis (real observations) and global analysis (segment hypothesis). This double reading level makes the forceful point of the method.

- this method can be used for grey level images as well as for binary images.

- moneover, when working with grey level images, our method (see section 3) does not imply a binarisation of this image to extract or nemove some layers.

\section{Bibliography}

[1] Baird, Bunke, Yanamoto (Eds), Structured Doctument Analysis. Springer Verlag, (1991)

(2) D. H. Ballard, C. M. Brown. Computer Vision. PrenliceHall, (1982).

[3] T. E. Fortmann, Y. Bar-Shalom, Tracking and data associdion. Mathematics in science and engincering, Yol. 179, Academic Press, (1988)

(4) V. Poulain d'Andecy, J. Camillerapp, I. Leplumey, Kalman filtering for segment delection : application to nus sic scores analysis.. 12th ICPR, Conputer Vision and Appitcations, Jerusalen, (9-13 ocloter 1994) Vol. 1; pp. 301-305.

[5] H. W. Sorenson, Kalnan fittering : Theory and Applica tion. IREE Press, (1985).

[6] J.P. Veran, Suivi de routes dans une image aerienne pur filtrage de Kalmat. Rappott technique de l'Ecole Nationale Supericute des Telecommunications, Departentent Images, Paris, (march 1993). 